\title{
Research on Identifying the Bottleneck of the Airport Security Inspection System Based on the Petri Net
}

\author{
Dongxu Chen
}

North China Electric Power University, Baoding 071000, China

e-mail:chendongxu0000@126.com

Keywords: Bottleneck, Petri Net, Markov Chain, Throughput

Abstract. The airport security inspection network model based on the Petri Net is established to identify the bottleneck of the airport security inspection system. A reach ability graph is set up to represent the process of the security inspection. We can get an isomorphic Markov Chain by simplifying the reach ability graph. Thus, the number of the token of each place is calculated by using the given data. As a result, the bottleneck is identified. We also calculated the throughput of each checkpoint by using the given data, the bottleneck is also identified. The bottlenecks found in the two ways are the same, showing that the model's accuracy is qualified.

\section{Introduction}

With the rapid development of the economy and society, airplanes now has become a more and more popular choice for modern people, especially for travelers and business men, due to its convenience and efficiency. As a result, airport security is very important. Usually, airports have security checkpoints, where passengers and their baggage are screened for explosives and other dangerous items. However, recently, some airlines came under sharp criticism for extremely long lines, and the long waiting time. Therefore, under the premise of ensuring the airport security, it is significant to find the bottleneck of the security inspection system.[1]

\section{Establishment of the Model}

Establishment of Petri Net with Time Parameter. To establish a stochastic Petri Net model with time parameters, we need to introduce a delay time which obeys the exponential distribution for each time transition of the system. According to the practical significance of the security system, in order to establish a Generalized Stochastic Petri Net (GSPN) model with instantaneous transitions of logical significance, an initiation probability should be determined for each instantaneous transition of the system. GSPN model of airport passenger security system is shown in Figure 1. [2][3][4]

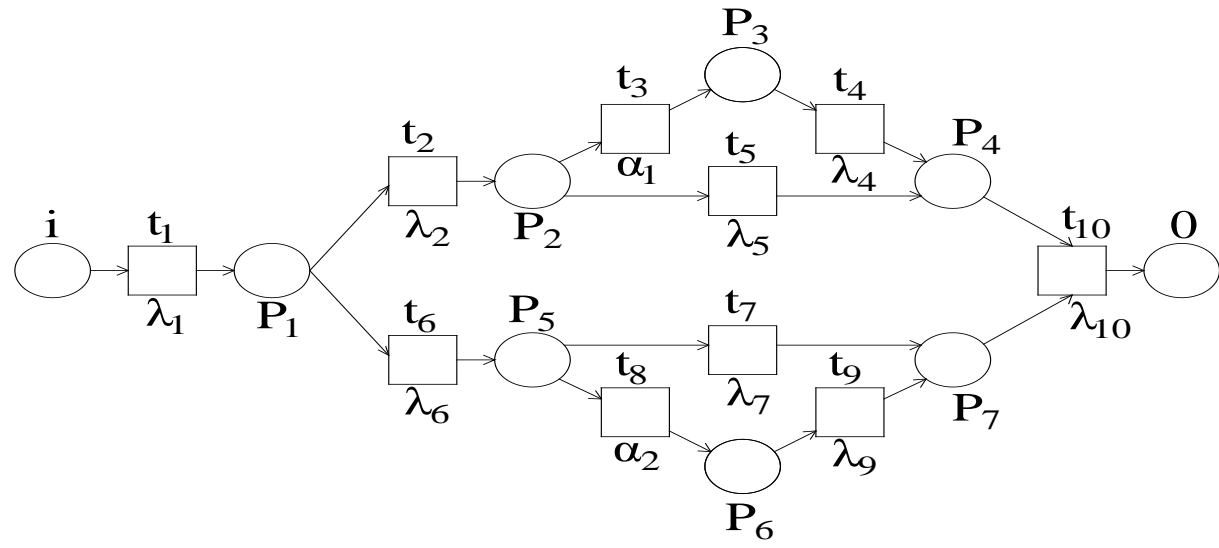

Fig.1 GSPN Model of Airport Security System

In the GSPN model, the set of time transitions:

$$
T_{a}=\left\{t_{1}, t_{2}, \cdots, t_{10}\right\}
$$

An initiation rate is determined for each time transition: 
The set of instantaneous transitions:

$$
\lambda_{a}=\left\{\lambda_{1}, \lambda_{2}, \cdots, \lambda_{10}\right\}
$$

$$
T_{a}=\left\{t_{3}, t_{5}, t_{7}, t_{8}\right\}
$$

If the token of $P_{2}$ exists, instantaneous transitionst $t_{3}, t_{5}$ are in enforceable state. If the probability of transition $t_{3}$ is $\alpha$, then the probability of transition $t_{3}$ is $1-\alpha$.

Construction of the MC with Isomorphism to GSPN. A stochastic Petri Net model is equivalent to a continuous-time Markov chain.[3][4]

Existence state

Disappearing state

$$
\left(M_{0}, M_{1}, M_{3}, M_{4}, M_{7}, M_{11}, M_{12}, M_{13}, M_{15}, M_{16}, M_{17}\right)
$$

After transitionst ${ }_{2}, t_{6}$,

$$
\left(M_{2}, M_{5}, M_{6}, M_{7}, M_{8}, M_{10}, M_{14}\right)
$$

Disappearing state $\left(M_{2}, M_{5}, M_{6}, M_{7}, M_{8}, M_{10}, M_{14}\right)$ is not considered.

We can obtain isomorphic MC by simplifying GSPN, as shown in Figure 2.

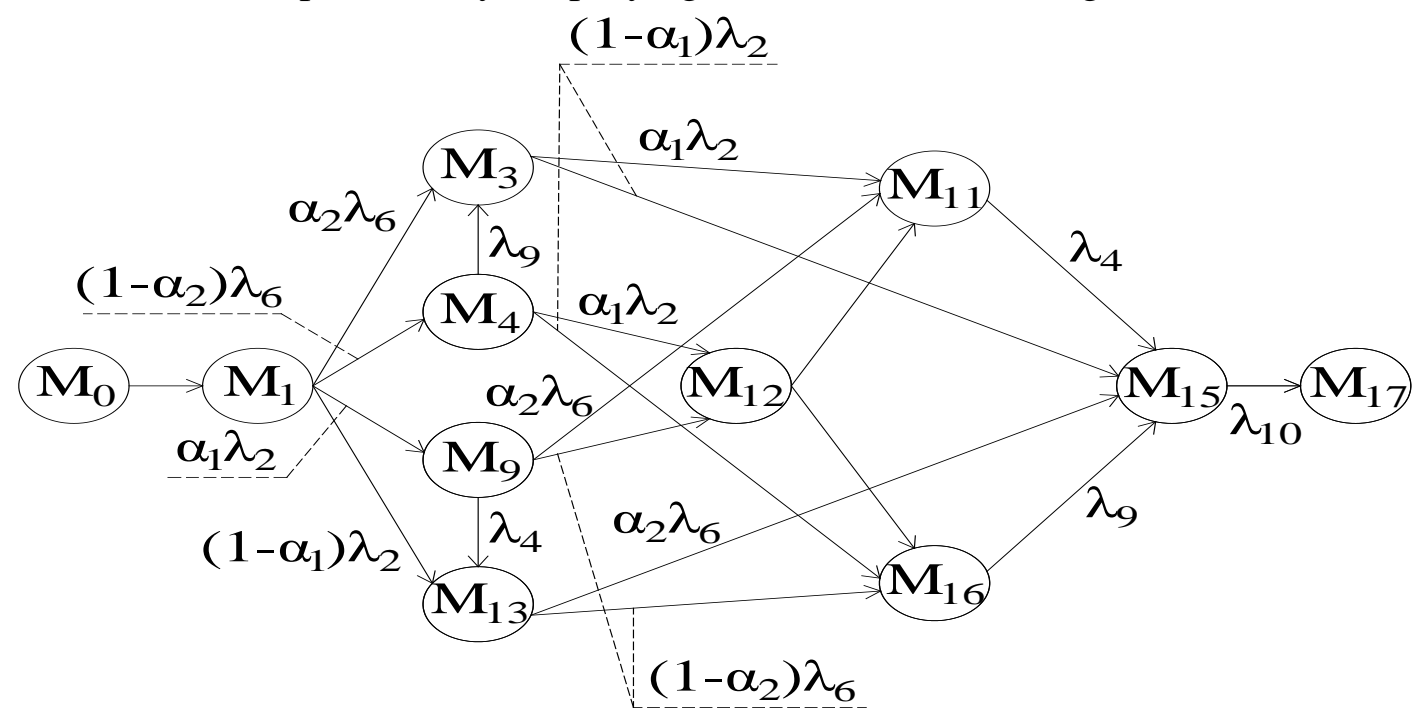

Fig.2 Isomorphic Markov Chain

Steady State Probability Distribution of Markov Chains. The probability of each state is:

$$
X=\left\{x_{0}, x_{1}, \cdots, x_{9}\right\}
$$

Using the density matrix $Q$, the probability distribution of the steady state is obtained by using the equation $X \cdot Q=0$.

\begin{tabular}{|c|c|c|c|c|}
\hline Checkpoint & The time consumption & $t_{i}$ & Initiation rate & Value \\
\hline ID Check & 12.53 & $t_{1}$ & $\lambda_{1}$ & 4.8 \\
\hline X-Ray & 7.54 & $t_{2}$ & $\lambda_{2}$ & 8.0 \\
\hline (No suspicious items) & - & 一 & 一 & - \\
\hline Millimeter Wave Scan & 11.64 & $t_{6}$ & $\lambda_{6}$ & 5.2 \\
\hline (No alarm) & - & - & - & - \\
\hline Gets canned property & 28.62 & $t_{10}$ & $\lambda_{10}$ & 2.1 \\
\hline
\end{tabular}

\section{Solution of the Model}

Taking the ordinary passenger as an example, according to the actual monitoring data at an airport in America, we can getTab.1: [3][4]

Table.1 Statistics of airport security system

According to the probability distribution of the steady state, the data in the Tab.1 is putted into the above model, then, the average number of tokens and vicissitude utilization of each place can be calculated, as shown in Tab. 2 . 
According to the Tab.2, we can get Figure 3:

Table.2 The average number of tokens and vicissitude utilization

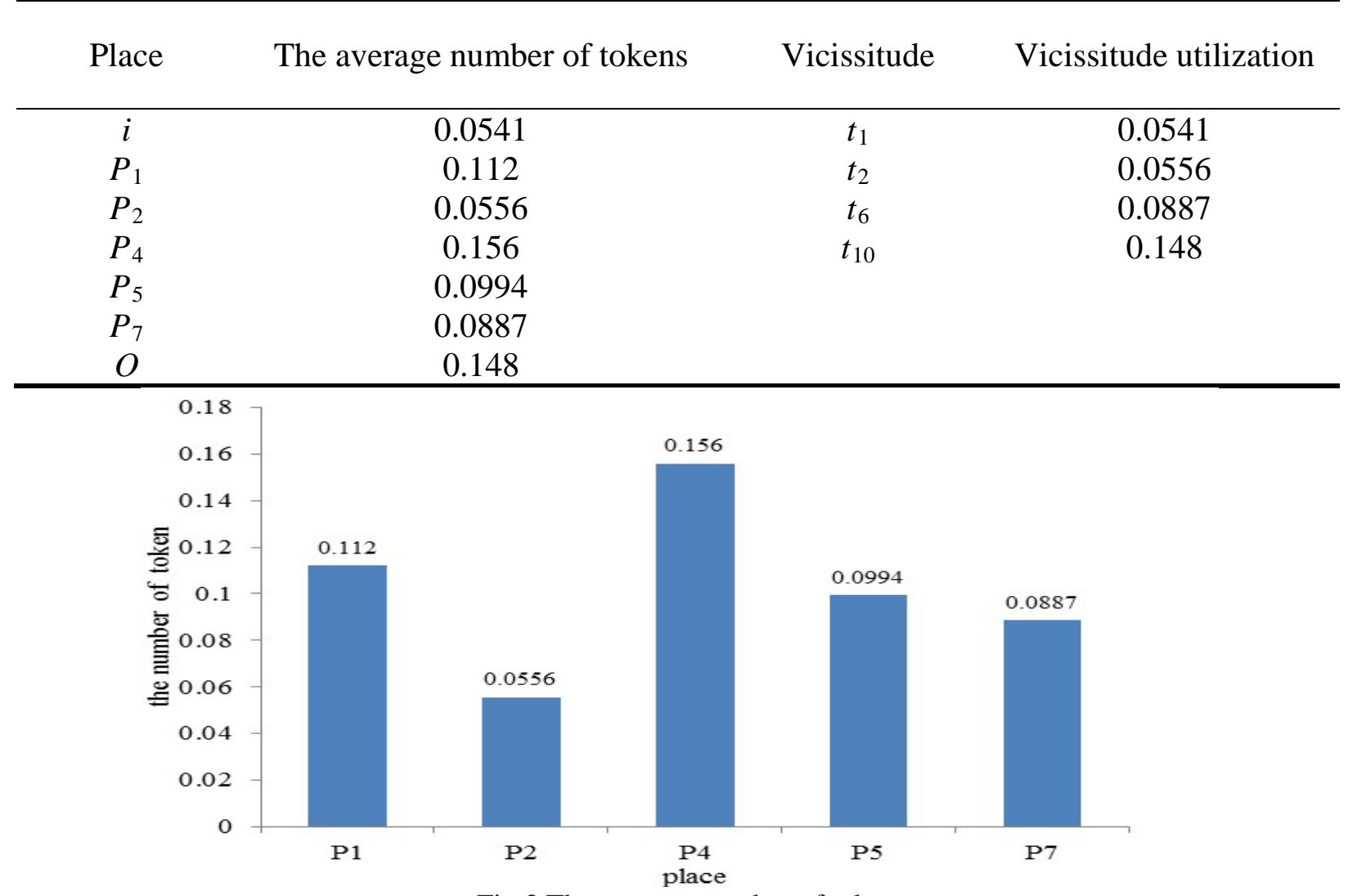

Fig.3 The average number of tokens

According to the Figure 3,the number of the token of the place $P_{4}$ is the largest, while $P_{2}$ is the smallest. $P_{4}$ is represented the checkpoint of getting scanned property, so we can conclude that the process of getting scanned property is the bottleneck of the security inspection lane.

\section{Model Validation Based on Data Processing}

The value of the passenger through put $R$ is calculated as follows: [5]

$$
R=\frac{n}{t}
$$

Where, $R$ is the passenger throughput at the checkpoint; $n$ donates the number of passengers passing a checkpoint over a period of time; $t$ is passenger pass time

Single-Channel Data Analysis. Each waiting time for regular lane and Pre-check lane is shown in Figure 4.

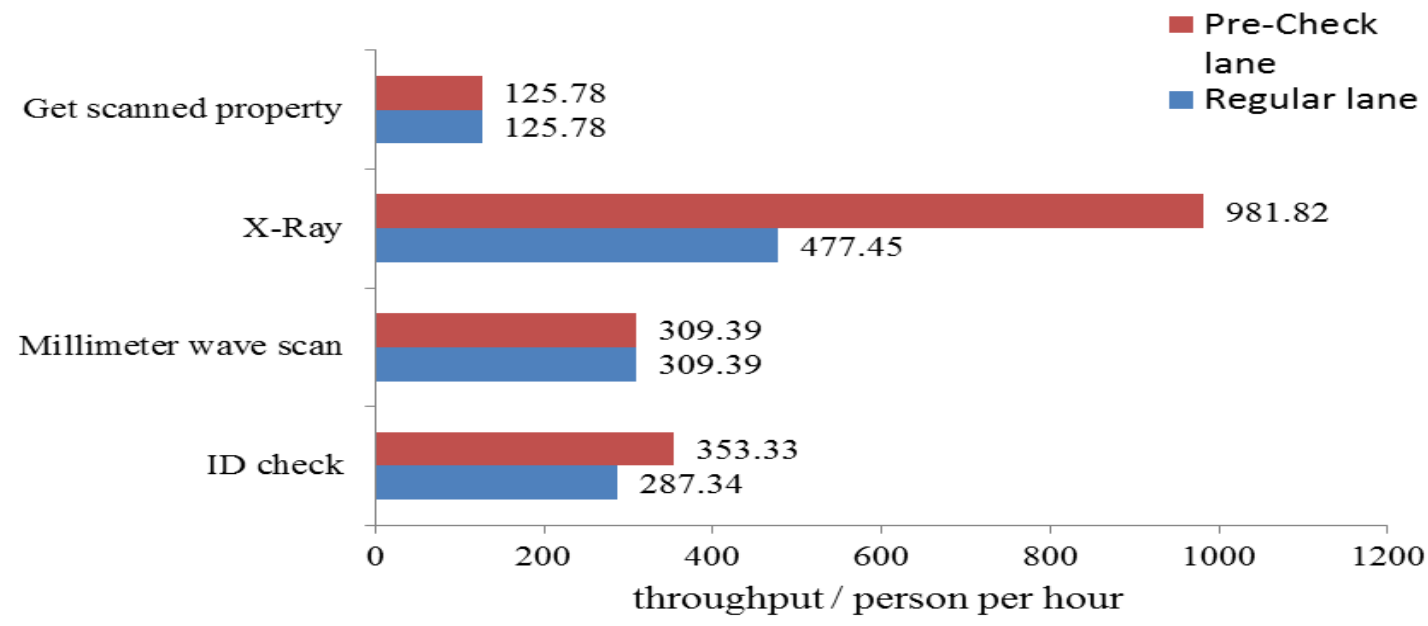

Fig.4 Each waiting time for regular lane and Pre-check lane 
According to the Figure 4, we can see: as for two kinds of lanes, the checkpoint for getting scanned property is the site where the passenger throughput is the smallest, which means that getting scanned property is the bottleneck of the lane.

Comparison between Pre-Check Lane and Regular Lane. Define bottleneck coefficient $K$, the expression of which is as follows:

$$
K=\frac{1}{R}
$$

Where, $R$ is the passenger throughput.

By calculation, we can get Tab.3:

Table.3 The value of bottleneck coefficient

\begin{tabular}{ccc}
\hline Lane & Regular Lanes & Pre-Check Lane \\
\hline Bottleneck coefficient & 0.00174 & 0.00323 \\
\hline
\end{tabular}

\section{Conclusion}

As the Tab.3 shown, the bottleneck coefficients of the regular lanes are less than the Pre-Check lane's. And therefore, in terms of the entire airport security, if there are too many trusted travelers in the airport, the Pre-Check lane is the bottleneck of the airport security. By comparing the results of each other, it can be seen that the conclusions of the two models are consistent. The main bottleneck of the two lanes is the process of getting scanned property. The above analysis shows that our model is correct.

\section{Reference}

[1] Qirui Han, "Research and Analysis of Airport Security Management System, "Yunnan University, 2015

[2] Jie Liu, Shengxue He, Haodong Zhang, et al, "Optimization Analysis on Sequence Bottleneck System of Urban Rail Transit,”ComputerApplication,January2016, Pages 271-274+286.

[3] Xinxin Jiang, Hang Zhou, Bing qing Cai, "Study on Terminal Layout and Process Optimization,”Aeronautical Computing Technology, March 2015, Pages 25-29+34.

[4] Yaping Zhang, Guoyang Jia, Shaowu Cheng, “Terminal Screening Process Modeling and PerformanceAnalysis Based on Petri Net, "Journal of Wuhan University of Technology (Transportation Science and Engineering), August 2015, Pages 688-691+697.

[5] Huangyang You, Lunhui Xu, "Research on Signal Timing of Bottleneck Intersection Based on Queuing Tension Coefficient, "Highways and Automotive Applications, July 2012, Pages 60-63. 